\title{
High neutrophil-to-lymphocyte ratio predicts early neurological deterioration in spontaneous intracerebral hemorrhage patients
}

Wafaa S. Mohamed ${ }^{*}$ D, Amr E. Kamel, Ahmed H. Abdelwahab and Mohamed E. Mahdy

\begin{abstract}
Background: Intracerebral hemorrhage $(\mathrm{ICH})$ is caused by bleeding, primarily into parenchymal brain tissue, and accounts for 9 to 27\% of all strokes worldwide. Higher neutrophils, lower lymphocytes, and higher neutrophil-tolymphocytes ratio (NLR) values predict worse outcomes after spontaneous intracranial hemorrhage (sICH) and could aid in the risk stratification of patients.

Methods: Eighty patients with slCH within the first $24 \mathrm{~h}$ of stroke onset and admitted into the neurology intensive care unit of an Egyptian university hospital and were assessed by GCS for consciousness level and NIHSS for stroke severity assessment, complete blood count, and special attention to NLR. Patients were reevaluated by GCS and NIHSS on the 7th day of the stroke. Early neurological deterioration (END) was defined as four points or a greater increase in the NIHSS score or two points or a greater decrease in GCS or death.

Results: END was recorded in $21.25 \%$ of patients while non-END was recorded in $78.75 \%$. END was highly significantly associated with a low grade of GCS, high grade of NIHSS, elevated absolute lymphocyte count (ALC), and elevated NLR. Lower GCS score, higher NIHSS score, larger hematoma volume, and higher NLR values were independent predictors for END. The best cutoff of NLR in END prediction was > 9.1.
\end{abstract}

Conclusion: NLR is a trustworthy early predictor of sICH outcome.

Keywords: Intracerebral hemorrhage, Neutrophil-to-lymphocyte ratio, Outcome

\section{Introduction}

Spontaneous intracerebral hemorrhage $(\mathrm{sICH})$ accounts for about 10 to $15 \%$ of all cerebrovascular strokes and affects over one million people per year globally. It is characterized by high rates of mortality and residual disability among survivors, with no therapeutic strategies of definitive benefit [1]. Early neurological deterioration (END) is common after sICH. It is associated with increased length of in-hospital stay, poor functional recovery, and death [2].

\footnotetext{
* Correspondence: Dr.wafaa74@yahoo.com

Department of Neurology, Faculty of Medicine, Zagazig University, Sharkia, Egypt
}

\section{Springer Open}

The immune reaction is a key characteristic of $\mathrm{ICH}$ pathology and affects its course. The response to cerebral hematoma is not limited to the brain and results in systemic effects. Inflammatory markers on admission, such as elevated leukocyte count, interleukin-6, and Creactive protein (CRP) are associated with poor prognosis [3-5]. The neutrophil-to-lymphocyte ratio (NLR) is linked to 3 months recovery in ICH patients [6], but its link with short-term outcome is undiscovered (Fig. 1). So we aimed to assess the association between leukocyte count and NLR on admission with the occurrence of END during the initial week after sICH onset.

(c) The Author(s). 2021 Open Access This article is licensed under a Creative Commons Attribution 4.0 International License, which permits use, sharing, adaptation, distribution and reproduction in any medium or format, as long as you give appropriate credit to the original author(s) and the source, provide a link to the Creative Commons licence, and indicate if changes were made. The images or other third party material in this article are included in the article's Creative Commons licence, unless indicated otherwise in a credit line to the material. If material is not included in the article's Creative Commons licence and your intended use is not permitted by statutory regulation or exceeds the permitted use, you will need to obtain permission directly from the copyright holder. To view a copy of this licence, visit http://creativecommons.org/licenses/by/4.0/. 


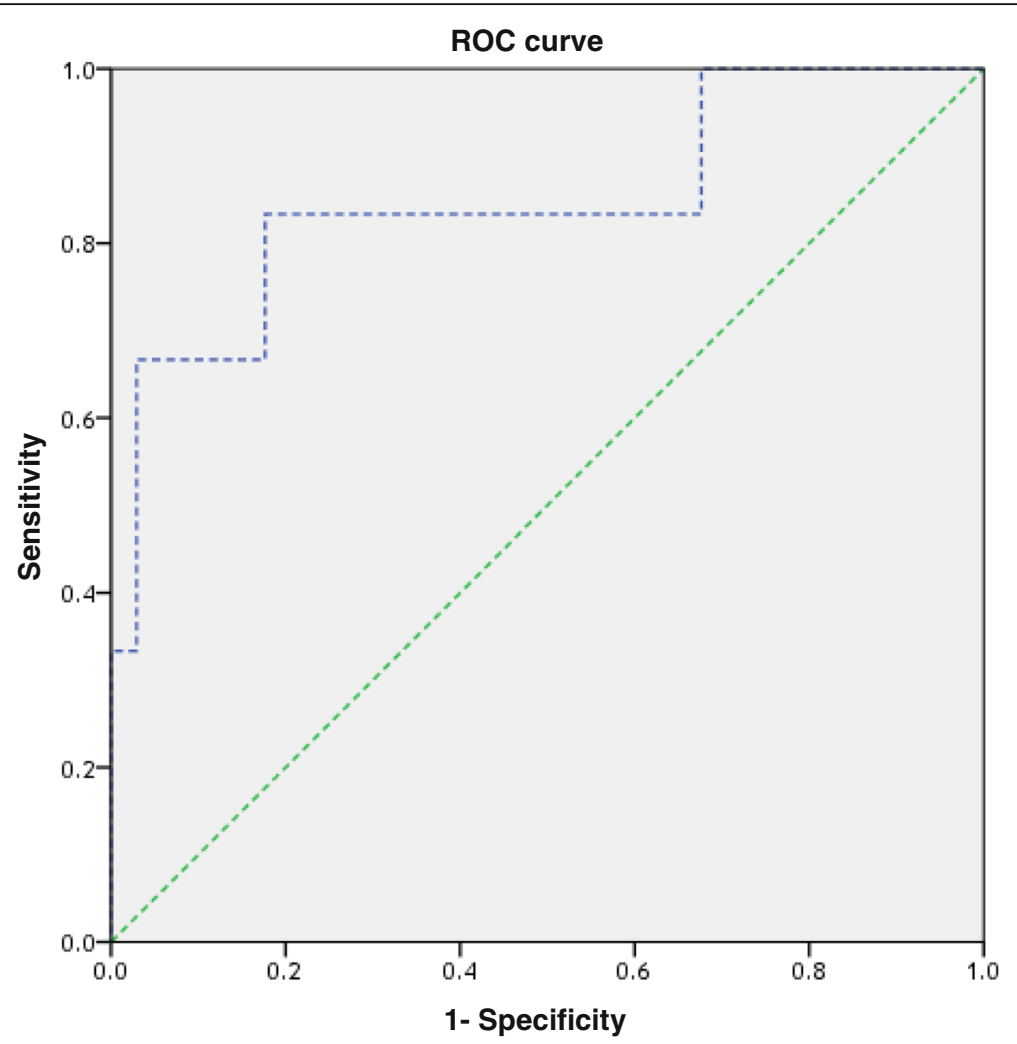

Fig. 1 ROC curve for detection of NLR cutoff with regard to END

\section{Patients and methods}

This prospective cohort study included 80 patients diagnosed with $\mathrm{sICH}$ within the first $24 \mathrm{~h}$ of stroke onset and admitted into the neurology intensive care unit.

\section{Inclusion criteria}

All patients were enrolled if their aged above 18 years old with first-ever sICH.

\section{Exclusion criteria}

Exclusion criteria included those who suffered from isolated intraventricular hemorrhage as the majority of these cases are associated with an underlying microvascular cause [7], hemorrhage secondary to a brain tumor, dural venous sinus thrombosis, patients on anticoagulants, patients receiving immune-modulatory treatment (e.g., corticosteroids, azathioprine, methotrexate, other cytostatic, and biological agents as monoclonal antibodies) as these agents alter the immune response of patients, patients with a head injury and surgery within 4 weeks preceding the event, patients with pre-existing infections like tuberculosis and hemorrhagic diseases or blood malignancies, patients with a severe degree of hepatic or renal disease, and patients with chest or urinary tract infection.
Patients were subjected to the following:

A full history was taken, including hypertension, diabetes mellitus, dyslipidemia, and smoking. General and neurological examination with assessment of consciousness state on admission using the Glasgow Coma Scale (GCS) and classifying our patients into three categories: those with a score of 3-7 (severely disturbed consciousness), 8-13 (moderately disturbed consciousness), and more than 13 (mildly disturbed consciousness) [8] and using the National Institutes of Health Stroke Scale (NIHSS) for stroke severity assessment. The severity of the stroke can be categorized according to the points of the score into mild (1-4), moderate (5-15), severe (16-20), and extremely severe (21-42) [9].

\section{Laboratory investigations}

- Complete blood count with special attention to the total leukocytic count, absolute neutrophil count (ANC), absolute lymphocyte count (ALC), platelet count, and NLR (by dividing ANC by ALC).

- Blood glucose level and its correlation with the patient's history if he was a known diabetic or not, 
to identify patients with admission stress hyperglycemia, liver and kidney functions, acute phase reactants especially CRP, and erythrocyte sedimentation rate (ESR).

\section{Radiological investigations}

- Computerized tomography (CT): using GE ProSpeed Dual Slice F II CT with MX135 Tube. CT brain was done, for all patients, on admission with stress on the identification of the site of hematoma right or left hemispheric, supra- or infra-tentorial; the size of the hematoma is measured by the formula (equation $A \times B \times C \times$ $0.5)$ where $A$ and $B$ indicate the largest perpendicular diameters through the hyperdense area on the CT scan, and $C$ indicates the thickness of slice $(9 \mathrm{~mm})$, presence of surrounding edema, and intraventricular extension [10].

\section{Follow-up}

All patients were followed up to detect END as all patients were re-evaluated by GCS and NIHSS on the 7th day of the stroke. END was defined as four points or a greater increase in the NIHSS score or two points or a greater decrease in GCS or death or both [6].

\section{Statistical analyses:}

The data were coded and entered using the statistical package SPSS. The data were summarized using mean and SD and median and IQ range for quantitative data, and number and percentage for qualitative data. Student's $t$ test was used to assess statistical differences between the two groups of quantitative data. Nonparametric Mann-Whitney (MW) and Kruskal-Wallis (KW) tests were used for quantitative variables, which were not normally distributed. $P$ values less than or equal to 0.05 were considered statistically significant.

\section{Results}

We included in this prospective cohort study 80 adult patients with first-ever acute intracerebral hemorrhage, 61 males (76.25\%) and 19 females $(23.75 \%)$ with age ranged from 30 to 85 years. The mean age was $57.132 \pm 11.37$. Eighty-eight percent of our patients were hypersensitive, $17 \%$ were diabetic, $54 \%$ were dyslipidemic, $42 \%$ were current smokers, and the body temperature of studied patients was $37.04 \pm 0.25(\mathrm{M} \pm \mathrm{SD})$ with a median value of 37.0 and range $36.7-38.5$. The patients with END presented were $21.25 \%$ (17 patients) while patients with non-END were $78.75 \%$ (63 patients). END was highly significantly associated with low grades of GCS (3-7) $(p=0.000)$ and high grade of NIHSS $(>20)(p=$ $0.000)$ (Table 1$)$. There was a highly statistically significant higher absolute lymphocyte count (ALC) (median $=0.8$, range $0.5-1$ ) and NLR (median = 10.9, range 8.6-33.3) among END patients (Table 2). NLR had significantly positive correlation with SBP $(p=0.02)$, DBP $(p=0.01)$, temperature $(p=0.000)$, and random blood sugar (RBS) $(p=0.000)$, NIHSS $(p=0.049)$, and negative significant correlation with GCS $(p=0.008)$ (Table 3$)$. In multivariate logistic regression for independent predictors of END, we found that severe or moderate coma as assessed by GCS ( $p=0.000$ and 0.02 respectively), extremely severe stroke as assessed by NIHSS $(p=0.02)$, hematoma size $>20.5 \mathrm{~mm}^{3}(p=0.000)$, and NLR > $9.1(p=0.002)$ were independent predictors for END (Table 4).

\section{Discussion}

We found that $17(21.25 \%)$ patients had END while 63 (78.75\%) patients did not have END, and $85.71 \%$ of our patients had GCS score 3-7 and 23.5\% of patients had GCS score 8-13 on admission which had highly

Table 1 Association of early neurological deterioration (END) with Glasgow Coma Scale (GCS) and the National Institutes of Health Stroke Scale (NIHSS) scores

\begin{tabular}{|c|c|c|c|c|c|c|c|c|}
\hline & & & \multicolumn{2}{|c|}{ END $(N=17)$} & \multicolumn{2}{|c|}{ Non-END $(N=63)$} & \multirow[t]{2}{*}{$x^{2}$} & \multirow[t]{2}{*}{$P$} \\
\hline & & & $N$ & $\%$ & $N$ & $\%$ & & \\
\hline \multirow[t]{3}{*}{ GCS } & Severe (3-7) & $N=7$ & 6 & 85.71 & 1 & 14.28 & 21.77 & 0.000 \\
\hline & Moderate (8-13) & $N=34$ & 8 & 23.5 & 26 & 76.5 & & \\
\hline & Mild (> 13) & $N=39$ & 3 & 7.69 & 36 & 92.31 & & \\
\hline \multirow[t]{4}{*}{ NIHSS } & Mild (1-4) & $N=8$ & 1 & 12.5 & 7 & 87.5 & 20.9 & 0.000 \\
\hline & Moderate (5-15) & $N=34$ & 1 & 2.9 & 33 & 97.1 & & \\
\hline & Severe $(16-20)$ & $N=23$ & 6 & 26.1 & 17 & 73.9 & & \\
\hline & Extremely severe (21-42) & $N=15$ & 9 & 60 & 6 & 40.0 & & \\
\hline
\end{tabular}

END early neurological deterioration, GCS Glasgow Coma Scale, NIHSS National Institutes of Health Stroke Scale 
Table 2 Relation of early neurological deterioration (END) with laboratory parameters

\begin{tabular}{lllll}
\hline & END $(N=17)$ & Non-END $(N=63)$ & Mann-Whitney & $P$ \\
\hline White blood cells (WBCs) (median-range) & $8.8(5.6-28)$ & $9.4(2.6-18.2)$ & 0.2 & 0.84 \\
Absolute neutrophil count (ANC) (median-range) & $7.1(4.6-25.5)$ & $6.8(2.1-16.6)$ & 0.84 & 0.4 \\
Absolute lymphocyte count (ALC) (median-range) & $0.8(0.5-1)$ & $1(0.3-3)$ & 2.8 & 0.005 \\
Neutrophil-to-lymphocyte ratio (NLR) (median-range & $10.9(8.6-33.3)$ & $5.4(1.3-36.2)$ & 3.7 & 0.000 \\
Erythrocyte sedimentation rate (ESR) (median-range) & $8(3.8-25)$ & $10(2-72)$ & 0.62 & 0.54 \\
C-reactive protein (CRP) (median-range) & $0.34(0.44-385)$ & $3.4(0.23-86)$ & 0.58 & 0.56 \\
\hline
\end{tabular}

END early neurological deterioration

**highly significant difference

significant relationship with END outcome. The same results were obtained by $[11,12]$ who found that patient's outcome was significantly related to the level of consciousness as assessed by GCS. Also, Wang et al. [13] and Zis et al. [14] all determined that there is a statistically significant relation between death after ICH and the admission GCS score.

There was a highly significant statistical relation between END and NIHSS. Matched results were obtained by Cheung and Zou [15] and Mahdy et al. [16] who found that admission NIHSS had a statistically significant relationship with the 30-day deterioration. Essa et al. [17] and Christensen et al. [18] also found a significant relationship between mortality following ICH and higher admission NIHSS. Our study did not demonstrate significant relation between WBCs and END which was matched with Behrouz et al. [19] and El-Tallawy et al. [12] who found that admission leukocytosis had no significant relation to a poor outcome. Contrary to our results, Mahdy et al. [20] found that the elevated WBC count was statistically significantly related to overall mortality.

There was a positive correlation between NLR and admission NIHSS. This is matching with the results of Giede-Jeppe et al. [21] who found, in a study of 855 patients with $\mathrm{sICH}$, that patients with NLR $\geq$

Table 3 Correlation between our study parameters and neutrophil-to-lymphocyte ratio (NLR)

\begin{tabular}{lll}
\hline & $r$ & $p$ \\
\hline Age & 0.11 & 0.29 \\
Systolic blood pressure & 0.25 & 0.02 \\
Diastolic blood pressure & 0.26 & 0.01 \\
Temperature & 0.40 & 0.000 \\
Random blood sugar (RBS) & 0.39 & 0.000 \\
Glasgow Coma Scale (GCS) & 0.29 & 0.008 \\
National Institutes of Health Stroke Scale (NIHSS) & 0.20 & 0.049 \\
Hematoma volume & 0.14 & 0.21 \\
Erythrocyte sedimentation rate (ESR) & 0.02 & 0.34 \\
C-reactive protein (CRP) & 0.20 & 0.06 \\
\hline
\end{tabular}

*significant difference

**highly significant difference
4.66 showed significant associations with poorer neurological status as measured by the National Institute of Health Stroke Scale. The same results were obtained by Sun et al. [22] who found that higher admission NLR was associated with higher NIHSS scores in a prospective study of 352 patients with acute ICH. In the present study, the NLR values on admission did not have a statistically significant correlation with age. This is matching with the results of Wang et al. [23] and Wang et al. [24] who found that NLR on admission had no statistically significant relation to age. On the other hand, other results were in disagreement with ours [25], which found that age was detected in a linear correlation with NLR in a retrospectively study of 336 patients with sICH. These different results may be due to their larger sample size (336 patients), and they followed up patients up to 3 months after sICH onset. Also, there was a significant relation between END and NLR values measured on admission. This is in agreement with the results obtained by Luo et al. [26], as they concluded that NLR values in the sICH patients rise significantly compared to that in the control group. This is also in agreement with the results obtained by Wang et al. [24] who found that the early rise of NLR predicts 30-day mortality in patients with sICH. Matching results were also obtained by Lattanzi et al. [27] who found that NLR was significantly associated with END during the first week after ICH onset.

\section{Conclusion}

There are different early predictors of sICH outcome which can be classified into clinical predictors (GCS and NIHSS), laboratory predictors (NLR), and radiological predictors (large hematoma volume).

\section{Recommendation}

Neurologists and ICU specialists should consider adding NLR values into consideration when predicting neurological deterioration in patients with sICH, in addition to studying this point on a larger sample size in other stroke units. 
Table 4 Multivariate logistic regression for independent predictors of early neurological deterioration (END)

\begin{tabular}{|c|c|c|c|c|}
\hline \multirow[t]{2}{*}{ Predictors } & \multirow[t]{2}{*}{ OR } & \multicolumn{2}{|c|}{$95 \%$ C.I } & \multirow[t]{2}{*}{$p$} \\
\hline & & Lower & Upper & \\
\hline \multicolumn{5}{|l|}{ Glasgow Coma Scale (GCS) } \\
\hline Severe & 80 & 8.6 & 1320 & 0.000 \\
\hline Moderate & 3.9 & 1.05 & 20 & 0.02 \\
\hline Mild & 1.0 & & & \\
\hline \multicolumn{5}{|l|}{ National Institutes of Health Stroke Scale (NIHSS) } \\
\hline Mild & 1.0 & & & \\
\hline Moderate & 0.25 & 0.01 & 3.8 & 0.20 \\
\hline Severe & 2.1 & 0.20 & 20.5 & 0.42 \\
\hline Extremely severe & 9.0 & 1.00 & 90.0 & 0.02 \\
\hline Hematoma volume (cutoff > 20.5) & 8.541 & 2.14 & 17.524 & $0.000^{* *}$ \\
\hline Temperature( cutoff > 37 ) & 2.587 & 0.547 & 12.321 & 0.354 \\
\hline Random blood sugar (cutoff > 160) & 1.987 & 0.245 & 12.32 & 0.541 \\
\hline White blood cells (WBCs) (cutoff > 8.2) & 3.254 & 0.87 & 18.21 & 0.087 \\
\hline Absolute neutrophil count (ANC) (cutoff > 6.9) & 4.258 & 0.95 & 11.21 & 0.059 \\
\hline Neutrophil-to-lymphocyte ratio (NLR) (cutoff > 9.1) & 5.541 & 2.11 & 25.14 & $0.002^{*}$ \\
\hline C-reactive protein (CRP) (cutoff > 0.93 ) & 3.547 & 0.874 & 19.654 & 0.065 \\
\hline
\end{tabular}

\begin{tabular}{lllll}
\hline Area & Cutoff & $P$ & \multicolumn{2}{l}{$95 \%$ Confidence Interval } \\
\cline { 3 - 5 } & & & Lower Bound & Upper Bound \\
\hline 0.848 & $>9.1$ & $0.007^{*}$ & .646 & 1.000 \\
\hline
\end{tabular}

The best cutoff of NLR in END prediction was > 9.1 and that had a highly significant value $(p=0.007)$

\section{Abbreviations}

sICH: Spontaneous intracerebral hemorrhage; END: Early neurological deterioration; NLR: Neutrophil-to-lymphocyte ratio; GCS: Glasgow Coma Scale; NIHSS: National Institutes of Health Stroke Scale; ANC: Absolute neutrophil count; ALC: Absolute lymphocyte count; ESR: Erythrocyte sedimentation rate; RBS: Random blood sugar

\section{Acknowledgements}

We acknowledge all participants of this research.

\section{Authors' contributions}

AEK, MEM, AHA, and WSM carried out this work. AEK designed the study, had done the statistical analysis, and wrote the manuscript. WSM and MEM did the literature search and coordinated the research team. MSM, WSM, and AHA collected the patients, gathered clinical data, and reviewed the manuscript. All authors were involved in drafting the article or revising it critically for important intellectual content, and all authors approved the final version to be published.

\section{Funding}

There is no source of funding for the research.

\section{Availability of data and materials}

Data and materials were available.

\section{Ethics approval and consent to participate}

The study was approved from the institutional ethics committee of Faculty of Medicine, Zagazig University (ZU-IRB\#4384/19-3-2018). A written consent was taken from all of the participants after explaining the details, benefits, as well as risks to them.
Consent for publication

Consent for publication has been obtained from the participants involved in the study to report their individual patient data.

\section{Competing interests}

The authors declare that they have no competing interests

Received: 31 March 2020 Accepted: 28 December 2020

Published online: 25 February 2021

References

1. Feigin VL, Lawes CM, Bennett DA, Anderson CS. Stroke epidemiology: a review of population-based studies of incidence, prevalence, and casefatality in the late 20th century. Lancet Neurol. 2003;2:43-53.

2. Mayer SA, Sacco RL, Shi T, Mohr JP. Neurologic deterioration in noncomatose patients with supratentorial intracerebral hemorrhage. Neurology. 1994:44:1379-84.

3. $\mathrm{Xi} \mathrm{G}$, Keep RF, Hoff JT. Mechanisms of brain injury after intracerebral hemorrhage. Lancet Neurol. 2006:5:53-63.

4. Castillo J, Dávalos A, Alvarez-Sabín J, Pumar JM, Leira R, Silva Y, et al. Molecular signatures of brain injury after intracerebral hemorrhage. Neurology. 2002;58:624-9.

5. Di Napoli M, Godoy DA, Campi V, Masotti L, Smith CJ, Parry Jones AR, et al. C-reactive protein in intracerebral hemorrhage: time course, tissue localization, and prognosis. Neurology. 2012;79:690-9.

6. Lattanzi S, Cagnetti C, Provinciali L, Silvestrini M. Neutrophil-to-lymphocyte ratio predicts the outcome of acute intracerebral hemorrhage. Stroke. 2016; 47:1654-7.

7. Hilkens NA, van Asch CJ, Rinkel GJ, Klijn CJ. Yield of angiographic examinations in isolated intraventricular hemorrhage: a case series and systematic review of the literature. Eur Stroke J. 2016;1(4):288-93.

8. Teasdale G, Jennett B. Assessment of coma and impaired consciousness: a practical scale. Lancet. 1974;304(7872):81-4

9. Adams H, Davis P, Leira E, Chang K-C, Bendixen B, Clarke W, et al. Baseline $\mathrm{NIH}$ Stroke Scale score strongly predicts outcome after stroke: a report of the Trial of Org 10172 in Acute Stroke Treatment (TOAST). Neurology. 1999; 53(1):126-31.

10. Kothari RU, Brott T, Broderick JP, Barsan WG, Sauerbeck LR, Zuccarello M, et al. The ABCs of measuring intracerebral hemorrhage volumes. Stroke. 1996;27(8):1304-5. 
11. Nilsson OG, Lindgren $A$, Brandt L, Säveland H. Prediction of death in patients with primary intracerebral hemorrhage: a prospective study of a defined population. J Neurosurg. 2002;97(3):531-6.

12. El-Tallawy H, Shawky OA, Farghaly S, Aziz ASA, Mahmoud Ashry ME. Predictive value of clinical presentation, laboratory parameters and $\mathrm{CT}$ brain findings of acute spontaneous intracerebral hemorrhage. Egypt I Neurol Psychiatry Neurosurg. 2005:42(1):177-85.

13. Wang YC, Zhou Y, Fang H, Lin S, Wang PF, Xiong RP, et al. (2014): Toll-like receptor $2 / 4$ heterodimer mediates inflammatory injury in intracerebral hemorrhage. Ann Neurol. 2014;75(6):876-89.

14. Zis $P$, Leivadeas $P$, Michas $D$, Kravaritis $D$, Angelidakis $P$, Tavernarakis $A$. Predicting 30-day case fatality of primary inoperable intracerebral hemorrhage based on findings at the emergency department. J Stroke Cerebrovasc Dis. 2014;23(7):1928-33.

15. Cheung RTF, Zou L-Y. Use of the original, modified, or new intracerebral hemorrhage score to predict mortality and morbidity after intracerebral hemorrhage. Stroke. 2003;34(7):1717-22.

16. Mahdy ME, Ghonimi NA, Elserafy TS, Mahmoud W. The NIHSS score can predict the outcome of patients with primary intracerebral hemorrhage. Egypt J Neurol Psychiatry Neurosurg. 2019;55:21.

17. Essa A, Helmy T, El Batch S. Study of incidence, risk factors and outcome of acute cerebrovascular stroke patients admitted to Alexandria Main University Hospital. J Am Sci. 2011;7(11):316-29.

18. Christensen MC, Morris S, Vallejo-Torres L, Vincent C, Mayer SA. Neurological impairment among survivors of intracerebral hemorrhage: the FAST Trial. Neurocrit Care. 2012;16(2):224-31.

19. Behrouz R, Hafeez $\mathrm{S}$, Miller CM. Admission leukocytosis in intracerebral hemorrhage: associated factors and prognostic implications. Neurocrit Care. 2015;23(3):370-3.

20. Mahdy ME, Zaitoun MA, Hasan HAEA, Aidaros MAEH. Predictors of the outcome in patients with primary intracerebral hemorrhage at Zagazig University Hospitals. In: A Thesis Submitted In Fulfillment of Medical Doctor Degree In Neurology. Egypt: Faculty of Medicine, Zagazig University; 2015.

21. Giede-Jeppe A, Bobinger T, Gerner ST, Sembill JA, Spruegel MI, Beuscher VD, et al. Neutrophil-to-lymphocyte ratio is an independent predictor for inhospital mortality in spontaneous intracerebral hemorrhage. Cerebrovasc Dis. 2017:44(1-2):26-34.

22. Sun Y, You S, Zhong C, Huang Z, Hu L, Zhang $X$, et al. Neutrophil to lymphocyte ratio and the hematoma volume and stroke severity in acute intracerebral hemorrhage patients. Am J Emerg Medicinex. 2015;35(3):429-33.

23. Wang F, Wang L, Jiang T-t, Xia J-j, X F F, Shen L-j, et al. Neutrophil-to-lymphocyte ratio is an independent predictor of 30-day mortality of intracerebral hemorrhage patients: a validation cohort study. Neurotox Res. 2018;34(3):347-52

24. Wang F, Xu F, Quan Y, Wang L, Xia JJ, Jiang TT, et al. Early increase of neutrophil-to-lymphocyte ratio predicts 30-day mortality in patients with spontaneous intracerebral hemorrhage. CNS Neurosci Ther. 2019:25(1):30-5.

25. Tao C, Hu X, Wang J, Ma J, Li H, You C. Admission neutrophil count and neutrophil to lymphocyte ratio predict 90-day outcome in intracerebral hemorrhage. Biomark Med. 2017;11(1):33-42.

26. Luo P, Li R, Yu S, Xu T, Yue S, Ji Y, et al. The relationship between neutrophil-to-lymphocyte ratio and intracerebral hemorrhage in type 2 diabetes mellitus. J Stroke Cerebrovasc Dis. 2017;27(5):930-7.

27. Lattanzi S, Cagnetti C, Provinciali L, Silvestrini M. Neutrophil-to-lymphocyte ratio and neurological deterioration following acute cerebral hemorrhage. Oncotarget. 2017;8(34):57489-94.

\section{Publisher's Note}

Springer Nature remains neutral with regard to jurisdictional claims in published maps and institutional affiliations.

\section{Submit your manuscript to a SpringerOpen ${ }^{\circ}$ journal and benefit from:}

- Convenient online submission

- Rigorous peer review

- Open access: articles freely available online

- High visibility within the field

- Retaining the copyright to your article

Submit your next manuscript at $\boldsymbol{\nabla}$ springeropen.com 